\title{
Predicting fadeout versus persistence of paratuberculosis in a dairy cattle herd for management and control purposes: a modelling study
}

\author{
Clara Marcé ${ }^{1,2,3}$, Pauline Ezanno ${ }^{1,2^{*}}$, Henri Seegers ${ }^{1,2}$, Dirk Udo Pfeiffer ${ }^{3}$, Christine Fourichon ${ }^{1,2}$
}

\begin{abstract}
Epidemiological models enable to better understand the dynamics of infectious diseases and to assess ex-ante control strategies. For Mycobacterium avium subsp. paratuberculosis (Map), possible transmission routes have been described, but Map spread in a herd and the relative importance of the routes are currently insufficiently understood to prioritize control measures. We aim to predict early after Map introduction in a dairy cattle herd whether infection is likely to fade out or persist, when no control measures are implemented, using a modelling approach. Both vertical transmission and horizontal transmission via the ingestion of colostrum, milk, or faeces present in the contaminated environment were modelled. Calf-to-calf indirect transmission was possible. Six health states were represented: susceptible, transiently infectious, latently infected, subclinically infected, clinically affected, and resistant. The model was partially validated by comparing the simulated prevalence with field data. Housing facilities and contacts between animals were specifically considered for calves and heifers. After the introduction of one infected animal in a naive herd, fadeout occurred in $66 \%$ of the runs. When Map persisted, the prevalence of infected animals increased to $88 \%$ in 25 years. The two main transmission routes were via the farm's environment and in utero transmission. Calf-to-calf transmission was minor. Fadeout versus Map persistence could be differentiated with the number of clinically affected animals, which was rarely above one when fadeout occurred. Therefore, early detection of affected animals is crucial in preventing Map persistence in dairy herds.
\end{abstract}

\section{Introduction}

In dairy herds, paratuberculosis, a worldwide disease caused by Mycobacterium avium subspecies paratuberculosis (Map), provokes decreases in milk production, drops in carcass slaughter value, and premature culling. It is important to predict as early as possible after Map first introduction in a dairy cattle herd whether infection is likely to fade out or to persist. This prediction could then be used to inform the implementation of control methods. Ideally, a point of no return should be identified, after which Map will persist and spread in the herd without control, i.e. when control actions must ideally be implemented. However, due to the long incubation

\footnotetext{
* Correspondence: pauline.ezanno@oniris-nantes.fr

'INRA, UMR1300 Bio-agression, Epidémiologie et Analyse de Risque en santé animale, BP 40706, 44307 Nantes, France

Full list of author information is available at the end of the article
}

period [1] and the low sensitivity of available diagnostic tests [2], studying the infection dynamics in the field is nearly impossible. Therefore, modelling is used to better understand Map spread within a herd.

Stochastic models are particularly suitable for investigating the likelihood of persistence versus fade-out of infection. Three stochastic models of Map transmission in dairy herds have been published [3-5]. However, these models neither take Map survival in the environment nor all relevant Map transmission routes into account, and therefore are not suitable for examining this persistence (see [6] for recent and detailed review of the models). Yet, the survival of Map in the environment can result in a delay between shedding by infectious animals and infection of susceptible animals. As a result of contamination of the farm environment,

C Biomed Central

C 2011 Marcé et al; licensee BioMed Central Ltd. This is an Open Access article distributed under the terms of the Creative Commons Attribution License (http://creativecommons.org/licenses/by/2.0), which permits unrestricted use, distribution, and reproduction in any medium, provided the original work is properly cited. 
infection of susceptible animals can occur in the absence of infectious animals $[7,8]$.

To study fadeout and persistence of Map in a dairy herd, we propose a new stochastic model that includes transmission via the environment. Furthermore, we have included calf-to-calf transmission, which has been demonstrated recently [9]. Hence, transmission routes are: vertical, horizontal via the ingestion of contaminated colostrum or milk, or horizontal via the ingestion of adult or calf faeces. Our model accounts for all of these transmission routes, thus rendering it possible to identify which routes contribute the most to Map spread in the modelled dairy herd. In the model, we assume that no further infected animals are introduced to avoid the possibility that persistence of Map might be due to continuous reintroductions (i.e. no fadeout being possible). Such a situation will be typical for herds with very low yearly purchase rates (e.g. dairy herds in Brittany without any fattening activity; [10]) or in the context of certification, when only certified animals are purchased (with a very low risk of being infected; [11]). In Europe, control of Map introduction into cattle herds has indeed priority over control of within-herd Map spread.

\section{Materials and methods}

We develop a model of Map spread within a dairy cattle herd initially naive towards Map infection, following the introduction of a single infected cow. We use this model to predict Map spontaneous fadeout or persistence as early as possible after Map introduction, before any control measure is implemented.

\section{Model description}

A discrete time compartmental model is developed to represent Map spread in a dairy cattle herd. We couple a model that simulates the population dynamics within a dairy herd and explicitly represents animal housing facilities with an epidemiological model of Map transmission. A time step of one week is chosen as the longest possible to allow the different transmission routes and calf exposure in housing facilities to be represented. A stochastic model is used in order to study the chance of fadeout of the disease versus persistence probability. Because of the slow progression of paratuberculosis, we choose to study the infection over a 25 -year period. The model is implemented with Scilab 5.1 [12].

\section{Population dynamics}

The population dynamics only considers characteristics related to Map transmission. Contacts between susceptible animals and any environment contaminated by shedding animals depends on the time spent by animals on farm, the time spent in individual and collective pens, and possible shared environments. An ageing process occurs before the infection process at each time step. An exit rate for mortality, sale, and culling is defined per age class (Table 1).

In Europe, dairy herds generally are structured in groups, the younger animals being separated from the older ones [13]. Here, group definition accounts for animal housing and management, and the maximal age $(u$, Table 2) at which an animal is susceptible (Figure 1). Therefore, contacts between susceptible animals and contaminated environments can be assessed. Calves younger than one year of age are either in individual pens (from birth to $m$ ), in collective pens before weaning (from $m$ to $w$ ), or in collective pens after weaning (from $w$ to $y$ ). Calves in individual pens have limited contacts with the faeces of calves from contiguous pens $(n b)$. Such a calf housing facility management follows European recommendations concerning animal welfare and social contacts (Council Directive 97/2/EC of 20 January 1997 amending Directive 91/629/EEC laying down minimum standards for the protection of calves ) and reflects the most common calf management in Europe [13]. After 1 year of age, the heifers are divided into 2 groups: from 1 year of age to $1^{\text {st }}$ artificial insemination (AI) at age $h$, and from $1^{\text {st }}$ AI to $1^{\text {st }}$ calving at age cal. Cows are all gathered in the same batch assuming they are not susceptible. Parities are considered as the culling rate is higher for older cows and to account for age in the progress of Map infection.

$X(a, t)$ represents the number of animals in health state $X$ and age $a$ at time $t$. Age is given in weeks until first calving (cal) and in parities (cal+1 to cal+5) after calving. An individual-based model is used until age $m$, when calves move to collective pens. Then, a compartmental model is used. If $a \leq m$, an index $k$ indicates in which individual pen the calf is: $X(a, t, k)=0$ or 1 depending on the occupancy of pen $k$. The total number of calves of age $a$ at time $t$ is:

$$
X(a, t)=\sum_{k=1}^{n} X(a, t, k) \text {, with } n \text { the number of individual }
$$

pens.

The herd model is calibrated by integrating knowledge from various sources, from published data to experts' knowledge, to realistically represent a French dairy cattle herd (Table 1). All male calves (half the calves) exit the herd during the $2^{\text {nd }}$ to $4^{\text {th }}$ week after birth (rate $\sigma_{m}$ ). Closed herds are modelled: there is no purchase of heifers for replacement. All female calves are thus kept to give flexibility to regulate the number of cows. Herd size is assumed to be stable over time. Heifers can be sold but only 10 weeks before the first calving (rate $\sigma_{h}$ ). Above a given number of cows $\left(K_{c}\right)$, the heifer sale rate increases. Under this threshold, the sale rate decreases. An all-year round calving is modelled with a mean 
Table 1 Parameters for herd management and population dynamics used in a Mycobacterium avium paratuberculosis infection dynamics model within a structured dairy herd

\begin{tabular}{|c|c|c|c|}
\hline Notation & Value & Definition & Source \\
\hline$\overline{\sigma_{B}}$ & 0.07 & Mortality rate of calves at birth & $\mathrm{a},[32]$ \\
\hline$\sigma_{m}$ & 0.206 & Exit rate of male calves, weeks 2 to 4 (per week) & \\
\hline$\sigma_{C 1}$ & 0.015 & Death rate of female calves, weeks 1 and 2 (individual housing facilities) (per week) & [32] \\
\hline$\sigma_{\subset 2}$ & 0.0035 & Death rate of female calves, weeks 3 to weaning (collective housing facilities) (per week) & [33] \\
\hline$\sigma_{C 3}$ & 0.00019 & Death rate of heifers from weaning to first calving (per week) & $\mathrm{b}$ \\
\hline$\sigma_{h}$ & 0.11 & Sale rate of bred heifers 10 weeks before $1^{\text {st }}$ calving & $b$ \\
\hline$\sigma_{A i}$ & $27,25,31,31,62$ & Yearly culling rate of cows in parity 1, 2, 3, 4 and above 5 respectively (\%) & $\mathrm{a},[34]$ \\
\hline$m$ & 2 & Maximal age in individual pen (weeks) & [13] \\
\hline w & 10 & Weaning age (weeks) & [13] \\
\hline y & 52 & Age when entering the young heifer group (weeks) & \\
\hline$n b$ & 2 & Number of neighbours for a calf in an individual pen & $b$ \\
\hline$h$ & 91 & Age at first artificial insemination (weeks) & a \\
\hline cal & 130 & Age at first calving (weeks) & $a, b$ \\
\hline$c c i$ & 56.3 & Calving-to-calving interval (weeks) & $a, b$ \\
\hline$b$ & 5 & Quantity of colostrum fed to calves ( $L /$ day for 3 days) & $\mathrm{b}$ \\
\hline$d$ & 7 & Quantity of milk fed to calves after 3 days (L/day/calf) & $\mathrm{b}$ \\
\hline prop & 0.85 & Proportion of lactating cows & a \\
\hline$\varepsilon$ & 25 & Quantity of milk or colostrum produced (L/day/cow) & a \\
\hline$f_{1}$ & 0.5 & Quantity of faeces produced by a non-weaned calf ( $\mathrm{kg} /$ day) & $\mathrm{b}$ \\
\hline$f_{2}$ & 5.5 & Quantity of faeces produced by a weaned calf ( $\mathrm{kg} /$ day) & $b$ \\
\hline$f_{Y}$ & 10 & Quantity of faeces produced by a heifer ( $\mathrm{kg} /$ day) & $\mathrm{b}$ \\
\hline$f_{A}$ & 30 & Quantity of faeces produced by a cow ( $\mathrm{kg} /$ day) & $\mathrm{b}$ \\
\hline Graz & {$[14-46]$} & Grazing period ( 1 being the first week of the year) & $b$ \\
\hline$K_{c}$ & 110 & Number of cows above which the heifer selling rate increases & - \\
\hline
\end{tabular}

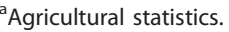

${ }^{\mathrm{b}}$ Expert opinions.

calving-to-calving interval $c c i$. Animals older than six months of age graze from April to November (Graz).

\section{Infection process and Map transmission}

The progression of individual animals through different Map infection states is a complex continuous process which is converted into discrete phases to enable the model parameterization based on current knowledge. Animals are classified into mutually exclusive health states: susceptible $(S)$, resistant $(R)$, transiently infectious $(T)$ (infectious only for a limited period of time), latently infected $(L)$ (infected not infectious), subclinically infected (Is) (infected and infectious but not affected), and clinically affected $(I c)$ (infected, infectious, and affected) [2]. Parameters are displayed in Tables 1 (herd dynamics), 2 (infection process), and 3 (shedding characteristics). Assumptions are based on current knowledge on Map.

Vertical transmission occurs with probability $p_{X}(T$ calf born to an infected cow). Horizontal transmission occurs by ingestion of colostrum, milk, or faeces. It depends on animal susceptibility, varying with age (maximal the first week of age and decreasing exponentially (h) until one year of age $(u)$ ). Under field conditions, animals older than one year of age have a low susceptibility to Map infection [14,15] and in the current model are therefore assumed to be resistant to infection. If infected, there is no possible recovery. We assume an exponential distribution of the durations in infection states $T, L, I s$, and $I c$. A transiently infectious state is assumed as infected calves have been reported to shed Map [9]. The transition from $T$ to $L$ either is modelled using a binomial distribution of probability $1 / v_{T}, v_{T}$ being the mean duration of the transiently infectious period, or occurs at the latest when the age at first calving ( $\mathrm{cal}$ ) is reached. A latent state is assumed because, if the absence of shedding has not been proven, the detection of infectious adults and heifers is hardly possible before animals reach one to two years of age, indicating at least quite a low level of shedding [16-18]. Latent animals are assumed not to shed Map, since shedding can be considered to be negligible compared with that of other infected adults. The transition from $L$ to Is is possible only after the $1^{\text {st }}$ AI (at age $h$ ). Subclinical animals are assumed to shed sufficient quantities of Map to be detectable and to contribute to Map spread within the herd, without having any obvious clinical 
Table 2 Parameters for infection and transmission used in a Mycobacterium avium subsp. paratuberculosis (Map) infection dynamics model within a structured dairy herd*

\begin{tabular}{|c|c|c|c|}
\hline Notation & Value & Definition & Source \\
\hline \multirow[t]{4}{*}{$p_{x}$} & & Probability of in utero transmission for cow in health state $X$ & {$[24,35]$} \\
\hline & $p_{L}=0.149$ & $X=$ latently infected $(L)$ & \\
\hline & $p_{/ s}=0.149$ & $X=$ subclinically infected $\left(I_{S}\right)$ & \\
\hline & $p_{K C}=0.65$ & $X=$ clinically affected $\left(I_{C}\right)$ & \\
\hline u & 52 & Maximal age in the susceptible compartment (weeks) & {$[15,36]$} \\
\hline$h$ & 0.1 & Susceptibility follows an exponential decrease $\exp (-h($ age- -1$)))$ & [14] \\
\hline \multirow[t]{5}{*}{$v_{X}$} & & Mean time spent in health state $X$ (weeks) & \\
\hline & $v_{T}=25$ & $X=$ transiently infectious $(T)$ & [9] \\
\hline & $v_{L}=52$ & $X=$ latently infected $(L)$ & {$[2,16]$} \\
\hline & $V_{15}=104$ & $X=$ subclinically infected $\left(I_{s}\right)$ & {$[37]$} \\
\hline & $v_{l c}=26$ & $X=$ clinically affected $\left(I_{C}\right)$ & a \\
\hline \multirow[t]{4}{*}{$\operatorname{sh}_{x}$} & & Probability of shedding in colostrum or milk for a cow in health state $X$ & {$[38,39]$} \\
\hline & $s h_{L}=0$ & $X=$ latently infected $(L)$ & \\
\hline & $s h_{15}=0.4$ & $X=$ subclinically infected $\left(I_{S}\right)$ & \\
\hline & $s h_{k c}=0.9$ & $X=$ clinically affected $\left(I_{C}\right)$ & \\
\hline$\alpha$ & $10^{6}$ & Map infectious dose & [40] \\
\hline$\beta_{l}$ & $5 \times 10^{-4} \times 7$ & Transmission rate if ingestion of an infectious dose (per week) & $\mathrm{b}$ \\
\hline$\beta_{c}$ & $5 \times 10^{-5} \times 7$ & Transmission rate if one infectious dose is present in the local environment (per week) & [9] \\
\hline$\beta_{g}$ & $9.5 \times 10^{-7} \times 7$ & Transmission rate if one infectious dose is present in the global environment (per week) & [9] \\
\hline$\beta_{\circ}$ & $5 \times 10^{-6} \times 7$ & Transmission rate if one infectious dose is present on pasture (per week) & $\mathrm{b}$ \\
\hline \multirow[t]{3}{*}{$g_{x}$} & & Decrease in milk production for cattle in health state $X$ (per week) & [41] \\
\hline & $g_{/ s}=2.5 \times 7$ & $X=$ subclinically infected $\left(I_{S}\right)$ & \\
\hline & $g_{k}=4 \times 7$ & $X=$ clinically affected $\left(I_{C}\right)$ & \\
\hline \multirow[t]{4}{*}{$\mu_{k}$} & & Removal rate of Map from environment $k$ & {$[7,8]$} \\
\hline & $\mu_{g}=0.4$ & all the environments (per week) & \\
\hline & $\mu_{i p}=0.67$ & individual pens (when empty) & \\
\hline & $\mu_{c p}=0.17$ & collective pens (when empty) & \\
\hline
\end{tabular}

*The values of the parameters in the epidemiological model (Table 2) are estimates based on experimental data reported in the literature. axpert opinions.

${ }^{\text {b}}$ Parameters' values are assumed.

signs. The transitions from $L$ to $I s, I s$ to $I c$, and $I c$ to exit of the herd are modelled using binomial distributions of probabilities $1 / v_{X}(X=L, I s$, or $I c), v_{X}$ being the mean time spent in state $X$. There is no additional mortality for $I s$ and $I c$ cattle, but $v_{I c}$ accounts for additional culling.

Depending on their age, $S$ calves are not all exposed to the same transmission routes. Calves born to infected dams can be infected via colostrum ingestion in the first week of age. During the first two weeks, calves are housed in individual pens. They can be infected via milk ingestion, exposure to the environment of the whole farm (global environment), or indirect transmission from infected calves of neighbouring pens. Before weaning, calves housed collectively can be infected via milk ingestion, exposure to the local environment of their pens, or exposure to the global environment. Inside (during winter), weaned calves can be infected via exposure to the local or to the global environment. On pasture, they can only be infected via exposure to the local environment shared with young heifers.

Colostrum and milk contamination occurs because of direct shedding or indirect faecal contamination. A calf ingests the colostrum of its dam. A calf $k$ born to a cow in state $X \in\{I s, I c\}$ ingests at time $t$ the following amount of bacteria:

$$
q_{c}^{k}=\operatorname{Bernouilli}\left(\operatorname{sh}_{X}\right)[f(X, \text { direct })+f(X, \text { indirect })] b
$$

with $f(X, r)$ the quantity of bacteria per litre of colostrum for an animal in state $X$ through route $r(f(X, r) \sim \mathrm{F}(X, r))$, $s h_{X}$ the probability of shedding in colostrum for cows in state $X$, and $\mathrm{b}$ the quantity of colostrum fed to calf. The number of calves infected via colostrum ingestion is then:

$$
\inf (c, t)=\sum_{k=1}^{k=n}\left[S(1, k, t) \text { Bernouilli }\left(1-\exp \left(-\frac{\beta_{l} q_{c}^{k}}{\alpha}\right)\right)\right](2)
$$




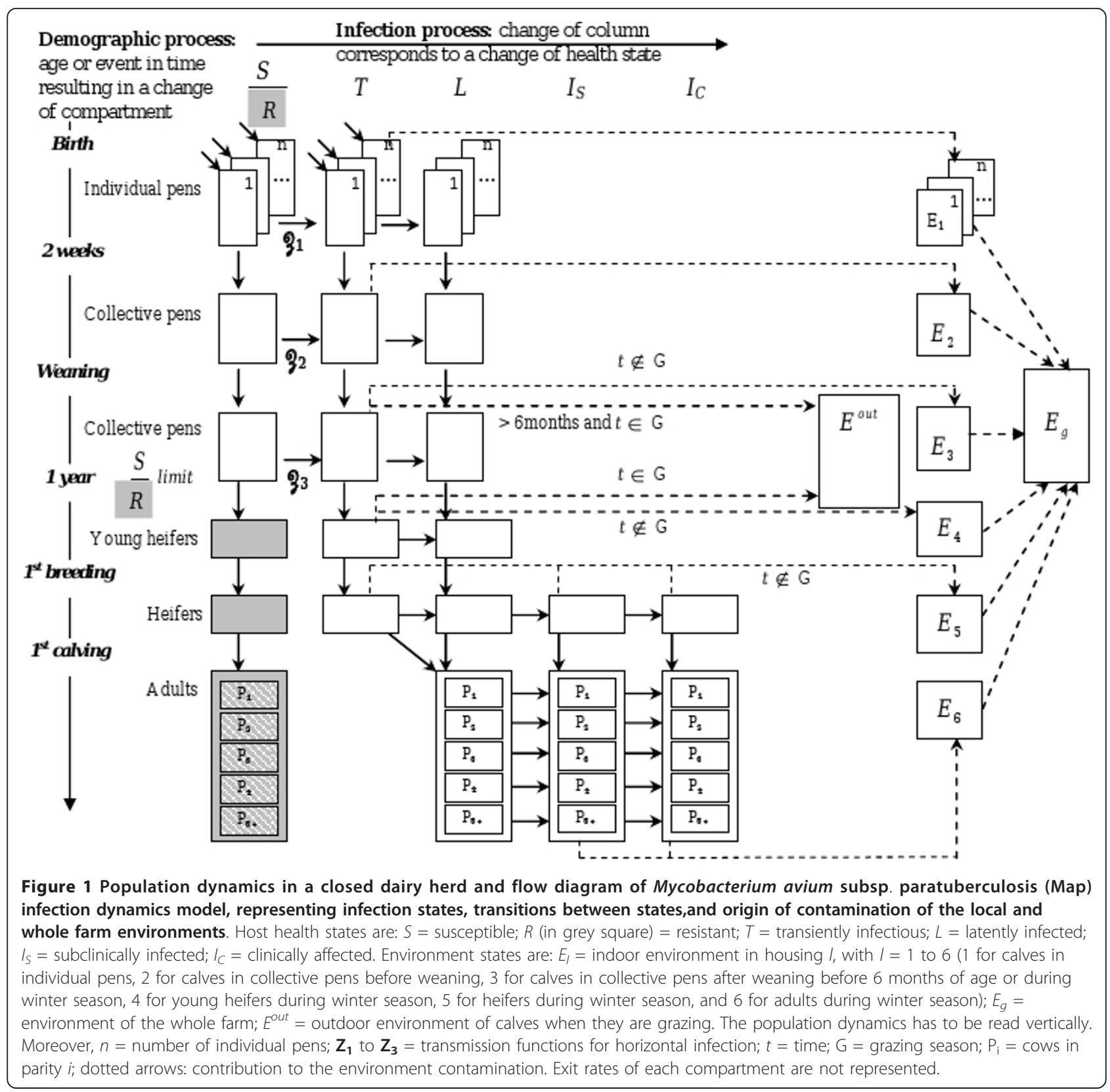

with $S(1, k, t)=1$ if there is a susceptible calf of one week of age in pen $k$ at time $t$ and 0 otherwise, $\beta_{l}$ the transmission rate if ingestion of an infectious dose, and $\alpha$ the infectious dose. Similarly, the number of calves infected via milk ingestion is:

$$
\inf (m, t)=\sum_{a=1}^{a=w}\left[\operatorname{Bin}\left(S(a, t), 1-\exp \left(-\exp [-h(a-1)] \frac{\beta_{l} q_{l}}{\alpha}\right)\right)\right]
$$

with $S(a, t)$ the number of susceptible calves of age $a$ at time $t, q_{l}$ the quantity of bacteria ingested per calf via milk ingestion. $q_{l}$ depends on the quantity of milk drank per calf $(d)$ and the quantity of bacteria in the tank, which depends on the proportion of $I c$ and $I s$ lactating (prop) and shedding $\left(s h_{X}\right)$ cows, these cows either directly shedding in milk $(f(X$,direct $))$ or because of faecal contamination of the milk $(f(X$, indirect $))$, and the quantity of milk they produce $\left(\varepsilon-g_{X}\right)$.

Faecal-oral transmission is indirect, occurring by ingestion of bacteria present in the environment. Two types of environment are modelled to differentiate indirect adult-to-calf from indirect calf-to-calf transmissions (Figure 1). $E_{g}$ is the quantity of Map in the global environment, contaminated by all of the shedding animals. 
It is assumed that all calves are equally exposed to the farm's environment, not accounting for possible variation in distribution of $M a p . E_{1}$ to $E_{3}$ are the quantities of $M a p$ in the calves' local environments, exclusively contaminated by $T$ animals housed in the associated facilities (Figure 1). We assume a homogeneous distribution of calves' faeces in a local environment or that all calves in a contaminated pen have the same probability of ingesting Map during a week. Susceptible animals are exposed to Map in the global and their local environments. The global environment is the sum of the local environments for calves and adults. All infectious cattle shed Map in their faeces. We assume shedding varies with the infection state, but also over time for a given infectious animal. We assume $T$ animals shed on average almost as much bacteria per $\mathrm{kg}$ of faeces as $I s$ animals but with a lower variability, Ic animals shedding much more (Figure 2). To model such a heterogeneity in shedding between animals and states, we fit distribution laws $\mathrm{F}(X$,faeces) (Figure 2) of Map quantities shed at time $t$ per kilogramme of faeces by a given animal of state $X$ to published observed data (Table 3). At time $t$, the quantity of Map per environment is updated, according to the removal rate $\mu$ (mortality of Map, cleaning of the barn, straw management) and Map shed by infectious animals. We assume no bacterium survives

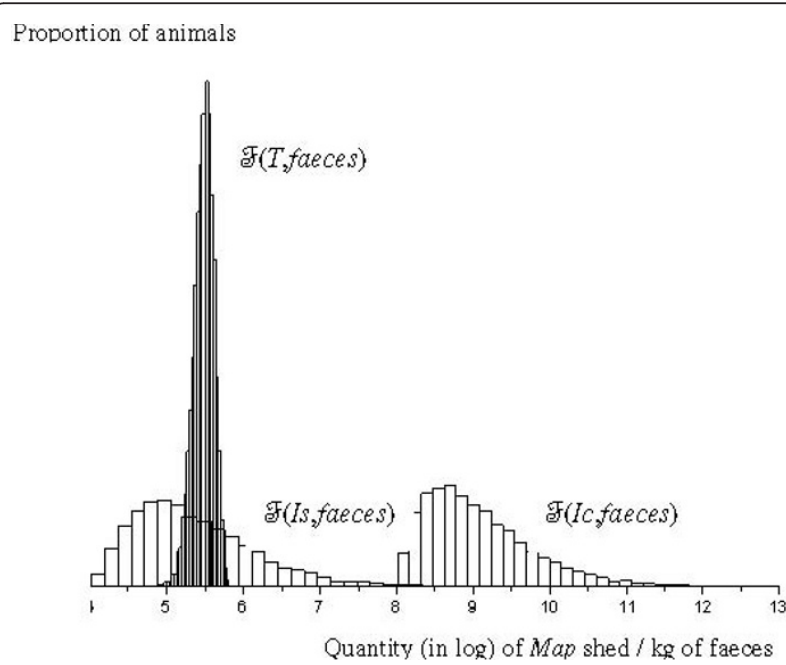

Figure 2 Distribution of the amount of Mycobacterium avium subsp.paratuberculosis (Map) shed per $\mathrm{kg}$ of faeces of transiently infectious $\left(F_{T}\right)$, subclinically infected $\left(F_{\mid s}\right)$ and clinically affected $\left(F_{\mid c}\right)$ animals used in the Map spread model within a dairy herd*. *Distributions are here in $\log ($ Map) $/ \mathrm{kg}$ of faeces (and not in Map/animal/day). Transiently infectious animals produce from 0.5 to $10 \mathrm{~kg}$ of faeces per day during 25 weeks on average $\left(f_{1}, f_{2}, f_{y}\right)$, whereas $/ s$ and $/ c$ animals are cows producing 30 $\mathrm{kg}$ of faeces per day $\left(f_{A}\right)$ for a longer period of time (Tables 1 and 2). Adults' contribution to total Map shed is thus more important than the one of transiently infectious animals. on pasture during winter; pastures are free of Map at next turn-out. In individual pen $k$, a susceptible calf of age $a$ is infected at time $t$ because of Map residuals in the pen with probability:

$$
P_{\mathrm{inf}}^{k}(a, t)=1-\exp \left(-\exp (-h(a-1)) \frac{\beta_{c} E_{l}(k, t)}{\alpha}\right)
$$

with $\beta_{c}$ the indirect calf-to-calf transmission rate.

Calves also can be infected because of their infectious neighbours (randomly sampled among calves). In collective pen $i$, susceptible calves of age $a$ are infected at time $t$ via calf-to-calf indirect transmission with probability:

$$
P_{\text {inf }}^{i}(a, t)=1-\exp \left(-\exp (-h(a-1)) \frac{\beta_{c} E_{i}(t)}{\alpha N_{i}(t)}\right)
$$

with $N_{i}(t)$ the number of animals in local environment $i$ at time $t$. Susceptible calves of age $a$ are infected at time $t$ via the global environment with probability:

$$
P_{\text {inf }}^{g}(a, t)=1-\exp \left(-\exp (-h(a-1)) \frac{\beta_{g} E_{g}(t)}{\alpha N(t)}\right)
$$

with $\beta_{g}$ the indirect transmission rate from this environment and $N(t)$ the herd size.

\section{Initial conditions}

All animals younger than $u$ are initially susceptible, other animals being resistant to infection. A subclinically infected parity one cow is introduced once in the herd with no further introduction. For each run, the date of introduction corresponds to the first week of January, i.e. three months before grazing starts. No specific measure is implemented in the herd to prevent or control Map infection. No change in herd management is implemented over time. Initially, study herds are composed of 277 animals (118 calves and young heifers, 45 bred heifers, and 114 cows).

\section{Model outputs}

Results are obtained from 400 runs over 25 years. We monitored the stability of means and variances of model outputs with increasing number of runs. We stopped when these estimates changed by less than $5 \%$ due to the last 100 runs. Therefore, runs are numerous enough to obtain stable simulated results. The first output is the infection persistence over time, i.e. the percentage of runs with the infection still present. We can deduce from this output the proportion of runs ending with fadeout. Other outputs then are studied separately for runs with persistent infection or runs with fadeout. The second output is the prevalence of infected $\left(T+L+I_{S}+I_{C}\right)$, infectious $\left(T+I_{S}\right.$ $\left.+I_{C}\right)$ and affected animals $\left(I_{C}\right)$ over time, these categories 
Table 3 Summary of published data and modelled distributions of the quantities of Mycobacterium avium subsp. paratuberculosis (Map) shed, depending on the health state $(\mathrm{X})$ and the route of transmission ( $\mathrm{r}$ ) in a Map infection dynamics model within a structured dairy herd

\begin{tabular}{|c|c|c|c|c|c|c|}
\hline \multirow[t]{2}{*}{ Route of transmission ( $r$ ) } & \multirow[t]{2}{*}{ Health state $(X)$} & \multicolumn{4}{|c|}{ Literature } & \multirow{2}{*}{$\begin{array}{l}\text { Model } \\
\mathrm{F}(X, \mathrm{r})\end{array}$} \\
\hline & & $\begin{array}{l}\text { Minimal } \\
\text { value }\end{array}$ & $\begin{array}{l}\text { Maximal } \\
\text { value }\end{array}$ & $\begin{array}{l}\text { Mean } \\
\text { value }\end{array}$ & Source & \\
\hline \multirow[t]{2}{*}{ Map direct shedding in milk and colostrum (Map/L) } & Subclinically infected & $2.2 \times 10^{4}$ & $8.8 \times 10^{4}$ & $5 \times 10^{4}$ & {$[39]$} & $10^{5} \times \operatorname{beta}(8,8)$ \\
\hline & Clinically affected & - & - & $5 \times 10^{4}$ & {$[42]$} & $10^{5} \times \operatorname{beta}(8,8)$ \\
\hline \multirow{2}{*}{$\begin{array}{l}\text { Map indirect shedding in milk and colostrum } \\
\text { (faecal contamination) (Map/L) }\end{array}$} & Subclinically infected & 0 & $2 \times 10^{10}$ & 40 & {$[43,44]$} & $1+10^{3} \times \operatorname{beta}(1,25)$ \\
\hline & Clinically affected & 700 & $2 \times 10^{10}$ & $14 \times 10^{4}$ & {$[43,44]$} & $10^{(3+10 \times \operatorname{beta}(50,200))}$ \\
\hline \multirow[t]{3}{*}{ Map shedding in faeces (Map/kg) } & Transiently infectious & $6 \times 10^{4}$ & $6.3 \times 10^{5}$ & $3 \times 10^{5}$ & [9] & $10^{6} \times \operatorname{beta}(8.8,19)$ \\
\hline & Subclinically infected & $10^{4}$ & $10^{15}$ & $2.6 \times 10^{6}$ & {$[45]$} & $10^{(4+10 \times \operatorname{beta}(2.65,17))}$ \\
\hline & Clinically affected & $10^{8}$ & $10^{15}$ & $10^{10}$ & {$[26,46]$} & $10^{(8+10 \times \operatorname{beta}(2,17))}$ \\
\hline
\end{tabular}

being defined by Nielsen \& Toft [19]. For runs with persistent infection, the pseudo-equilibrium of the prevalence is estimated. Among the two types of runs, the proportion of animals that become $I_{C}$ or detected with a systematic test (sensitivity of 0.5 and specificity of 1 ) during the early infection dynamics is studied. The third output is the relative contribution of the transmission routes to the number of newly infected animals.

\section{Model evaluation}

First, model outputs are compared with published data and field data from infected herds [20,21]. The simulated proportion of infected adults is compared to the estimated prevalence of infected adults on farms that voluntarily participated in a control program based in Brittany (France) [22]. Data corresponds to 59 herds enrolled in the program between 2002 and 2005 and in which more than 20 adults per herd were tested in the year of enrolment. All adults older than 24 months of age were tested annually using both ELISA and either PCR or faecal culture until 2007, and systematic ELISA and PCR in faeces of ELISA positive animals in 2008. Ziehl-Neelsen tests [1] were performed when suspect clinical signs were observed. Individual statuses of adults during the first year of the program implementation (i.e. before any control measure was introduced) are retrospectively attributed based on a maximum of three successive annual results. These statuses are defined as: clinically affected (Ziehl-Neelsen positive test in the first year), subclinically infected (PCR or faecal culture positive in the first year but Ziehl-Neelsen negative if performed), latently infected (seropositive in the first year but PCR or faecal culture negative or negative in all tests in the first year with a positive test later, whatever the test), and resistant (testing negative in all tests). For animals always testing negative but with only one or two tests (instead of three), we assume that they are either resistant (optimistic option which may under-estimate infection) or latently infected (pessimistic option which may over-estimate infection). Based on these optimistic and pessimistic distributions, we estimate the distribution of animals per infection state at the start of the program and the withinherd prevalence at enrolment. To compare model outputs with field data, we assume farmers usually detect the disease from 5 to 9 years after Map introduction (time needed for clinical cases to occur). We calculate the distribution of the mean simulated prevalence in infected adults in infected herds over this time period.

Second, a hypothesis-testing approach is used to validate the model, assuming a constant herd structure. We verify that either our conclusions are robust to variation in model parameters, or that parameter variation induces unrealistic within-herd prevalence and therefore cannot be retained. A one-at-a-time sensitivity analysis is performed for uncertain parameters $\left(v_{T}, u, h, p_{x} s h_{x}\right.$ $\beta_{l}, \beta_{c}, \beta_{g}, \beta_{o}, \mathrm{~F}(T$,faeces $\left.)\right)$. Variations of $\pm 50 \%$ from reference values are tested where applicable $\left(v_{T}, u, h, p_{x}\right.$ $\left.s h_{x}, \beta_{l}, \beta_{c}, \beta_{g}, \beta_{o}\right)$. For $\mathrm{F}(T$,faeces $)$, the worst plausible case is tested, $T$ animals shedding (per kilogram of faeces) as much as Is animals, with the same variability.

Third, to evaluate how the conclusions change with herd management, a model evaluation is performed as regards to variations of parameters managed on farm $\left(\mu_{k}, K_{c}, v_{I c}, G r a z\right)$. Variations of $\pm 50 \%$ from nominal values are tested for $\mu_{k}$, and $v_{I c}$. For $K_{c}$ (closely related to herd size), limits of 50 vs. 500 cows are tested. Lastly, a delay in the start of grazing (same duration but starts in the week Map is introduced vs. ends in the week before Map is introduced) and a variation in its duration (same start but duration of 28 vs. 37 weeks) are tested.

\section{Results}

Spontaneous fadeout of Map infection without control measure

Spontaneous fadeout occurred in $66 \%$ of the runs (Figure 3). In $43 \%$ of the runs, it occurred within the first two years (early extinction), while it occurred less 


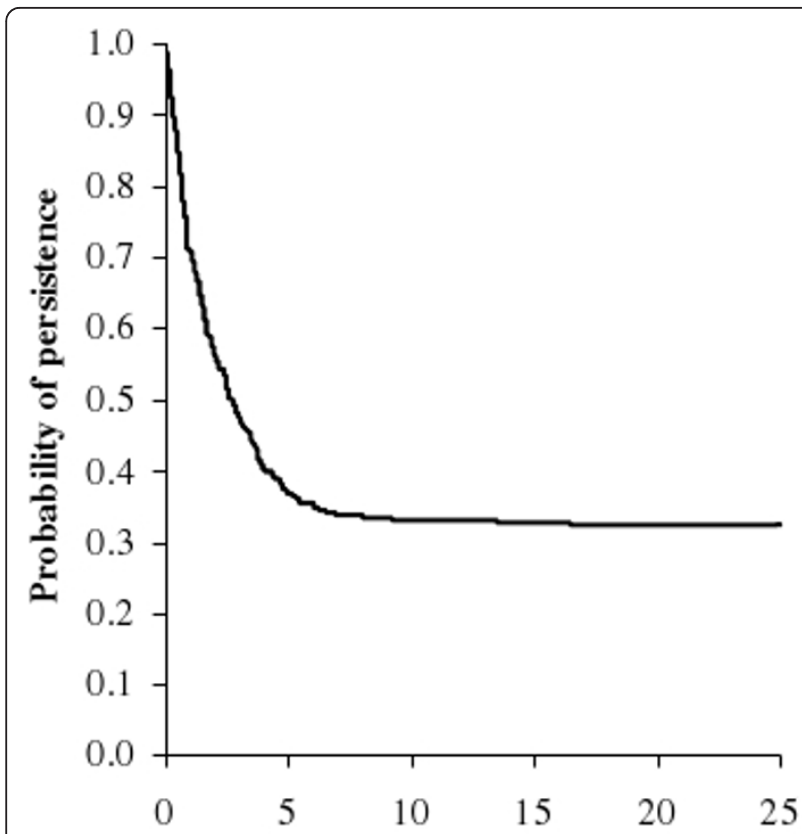

Time since Map introduction (in yrs)

Figure 3 Probability of persistence over time (proportion of runs where an infected animal is still present) of Mycobacterium avium subsp. paratuberculosis (Map) infection in a dairy cattle herd after a single Map introduction $(t=0)$ in the herd.

quickly in the remaining $23 \%$. Herds still infected seven years after Map introduction had thereafter a fadeout probability less than $6 \%$. When shedding animals were no longer present on the farm but the environment was still contaminated thus fadeout had not occurred yet, new infection of cattle from residual Map in the environment occurred with a mean weekly probability of $3 \%$. Hence, once the environment has been contaminated, spontaneous fadeout was hardly possible.

The probability of fadeout only slightly varied with uncertain parameters (from 62 to $71 \%$ ). It decreased to $51 \%$ when the mean time spent in state $I c$ increased by $50 \%$, and to $58 \%$ when Map removal from the global environment decreased by $50 \%$. Other parameters relating to herd management only had little influence on the fadeout probability.

It needs to be emphasized that yearly single introduction of Map would lead to a decrease in the cumulative probability of spontaneous fadeout, which can be calculated for $n$ years using $0.66^{n}$ (e.g. $66 \%$ the first year as in the present study, $44 \%$ the second year, $29 \%$ the third year, etc.).

\section{Map spread within persistently infected herds}

Prevalence of infection reached a pseudo-equilibrium (when accounting only for runs in which infection persisted) 23 years after Map introduction when no control measure was implemented (Figure 4). At the end of the simulation period, the prevalence of infected, infectious, and affected animals reached $88 \%, 44 \%$, and $6 \%$, respectively. In adults, prevalence of infected, infectious, and affected animals was $87 \%, 67 \%$, and $15 \%$, respectively. Annual incidence reached 15\% (Figure 4).

Comparing the simulated and the observed distributions of prevalence in infected herds indicated that the model over-estimated the cases when infected herds had a low prevalence (more than $40 \%$ of the infected runs had a prevalence in infected adults less than 5\%; Figure 5). For other levels of prevalence, simulated and observed distributions were similar.

Varying uncertain parameters produced in most cases $\left(u, v_{T}, p_{I o} s h_{X}, \beta_{o} \beta_{b} \beta_{o}, \mathrm{~F}(T\right.$,faeces $\left.)\right)$ prevalence distributions similar to the reference scenario and therefore these parameters cannot be more precisely estimated from the sensitivity analysis. For others $\left(h, p_{L}, \beta_{g}\right)$, a variation of $\pm 50 \%$ resulted in a simulated prevalence not consistent with the observed prevalence.

Among infected adults, the model provided mean proportions of $L, I_{S}$, and $I_{C}$ animals 25 years after Map introduction of 60,32, and 8\%, respectively (Figure 6A). These proportions slightly varied over time, except in the transient period when prevalence was very low. In field data (Figure 6B), the proportion of animals per infection state depended on the option: the pessimistic option resulted as expected in a large proportion of latently infected animals. The mean proportion of subclinically infected animals varied from 17 to $40 \%$ in the optimistic option, and from 3 to $22 \%$ in the pessimistic option. Simulation values were in between the two assumptions (Figure 6).
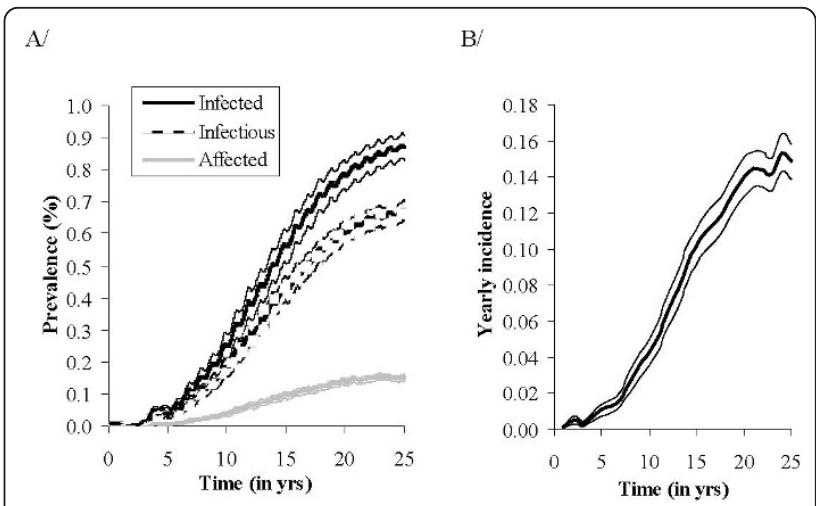

Figure 4 Mycobacterium avium subsp. paratuberculosis (Map) spread in a persistently infected dairy cattle herd since Map introduction $(\boldsymbol{t}=\mathbf{0})$. A/Mean prevalence over time of infected (black), infectious (dark grey), and affected (light grey) adults (> 30 months) and related confidence intervals. B/Mean annual incidence and related confidence interval. 


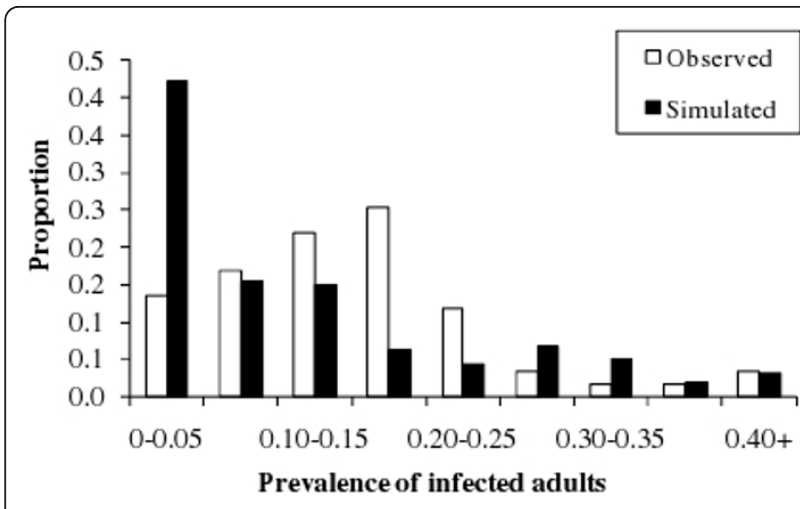

Figure 5 Comparison of the simulated and the observed distributions of the prevalence in Mycobacterium avium subsp. paratuberculosis (Map) infected adults in infected dairy cattle herds. The simulated distribution corresponds to runs of a Map spread model within a dairy cattle herd, the mean prevalence from year 5 to year 9 since Map introduction in the herd $(t=0)$ being calculated for each run still infected. The observed distribution is based on individual life long determined statuses in 59 dairy herds at enrolment in a paratuberculosis control program in France, before any control measure is implemented.

At the herd level, the main transmission routes were indirect transmission via the contaminated global environment, then in utero transmission. Transmission via colostrum or milk ingestion and calf-to-calf indirect transmission appeared to be minor routes (Figure 7). For high within-herd prevalence, the two main transmission routes equally contributed to new infections (Figure 7B). For parameter variation resulting in plausible results, these conclusions remained unchanged. Even an increase of one $\log (* 10)$ of the indirect transmission rate in the calf environment barely changed the contribution of calf-to-calf indirect transmission, which slightly increased for a low within-herd prevalence. Assuming $T$ animals shed as much as $I s$ animals (per kg of faeces) resulted in calf-tocalf indirect transmission contributing as much as in utero transmission for a very low within-herd prevalence, the contribution decreasing for a prevalence higher than $5 \%$.

\section{Characteristics of the runs ending in fadeout vs. persistent infection}

No secondary infection (on top of the first introduced case) was observed in $75 \%$ of the runs with fadeout, contrary to herds persistently infected. Only $3 \%$ of the runs ending with fadeout had at least two clinically affected animals (simultaneously or successively) over five years, compared to $80 \%$ of the persistently infected runs (Table 4). When combining clinical surveillance and systematic testing of cows, more than 2 animals were detectable after 3 years in $18 \%$ of the runs with fadeout and in $68 \%$ of the runs with persistent infection (21\% and 96\%, respectively after 5 years).
Based on the model outputs in Table 4, we can predict at the herd level the probability of Map persistence for a situation under a given detection threshold vs. the probability of spontaneous fadeout for a situation over this threshold. If a control programme based on clinical surveillance is implemented when at least one affected animal is observed in five years, the programme is unnecessarily implemented (fadeout would have spontaneously occurred) in $48 \%$ of the cases (i.e. the number of runs over the threshold ending with fadeout over the total number of runs over the threshold). If no control programme is implemented (no affected animals in five years after Map introduction), a persistent infection occurs in $1 \%$ of the cases. For a threshold of two affected animals, these proportions are $9 \%$ and $8 \%$, respectively. For a threshold of 3, they are $4 \%$ and $14 \%$, respectively. However, only $24 \%$ of the persistently infected herds had at least 2 affected animals within 3 years after Map introduction, $80 \%$ within 5 years. If the control programme is based on both clinical surveillance and imperfect tests (assuming a sensitivity of 0.5 and a specificity of 1) targeting adults, the proportions become $61 \%$ and $2 \%$ for at least 1 detected animal in 3 years after Map introduction, 39\% and 14\% for a threshold of 2 , and $5 \%$ and $19 \%$ for a threshold of $3.68 \%$ of the persistently infected herds had at least 2 detected animals within 3 years after Map introduction, 96\% within 5 years.

\section{Discussion}

The results from model experimentation have improved the understanding of Map spread within a dairy herd. Fadeout could occur even without implementation of control measures in an infected herd. This demonstrates the usefulness of a modelling approach, since such fadeout cannot be observed in the field given the low prevalence of infection and low likelihood of detection using available diagnostic methods. Probability of fadeout was estimated at $66 \%$, showing this probability can be high. This absolute value cannot be used directly as it cannot be validated with observed data since most fadeout events cannot be observed. It is likely to vary with model assumptions (including herd characteristics). Nevertheless, the sensitivity analysis demonstrated that fadeout is likely to be frequent in a wide range of situations. The economic assessment of paratuberculosis control programmes should therefore account for this high probability. Moreover, this model predicts changes in the fadeout probability when the delay to cull clinically affected animals varies and shows how important a control measure it is. This model can be used similarly to evaluate the relative impact of other interventions.

The cumulated number of clinically affected animals appears to be a good indicator of the progression of 

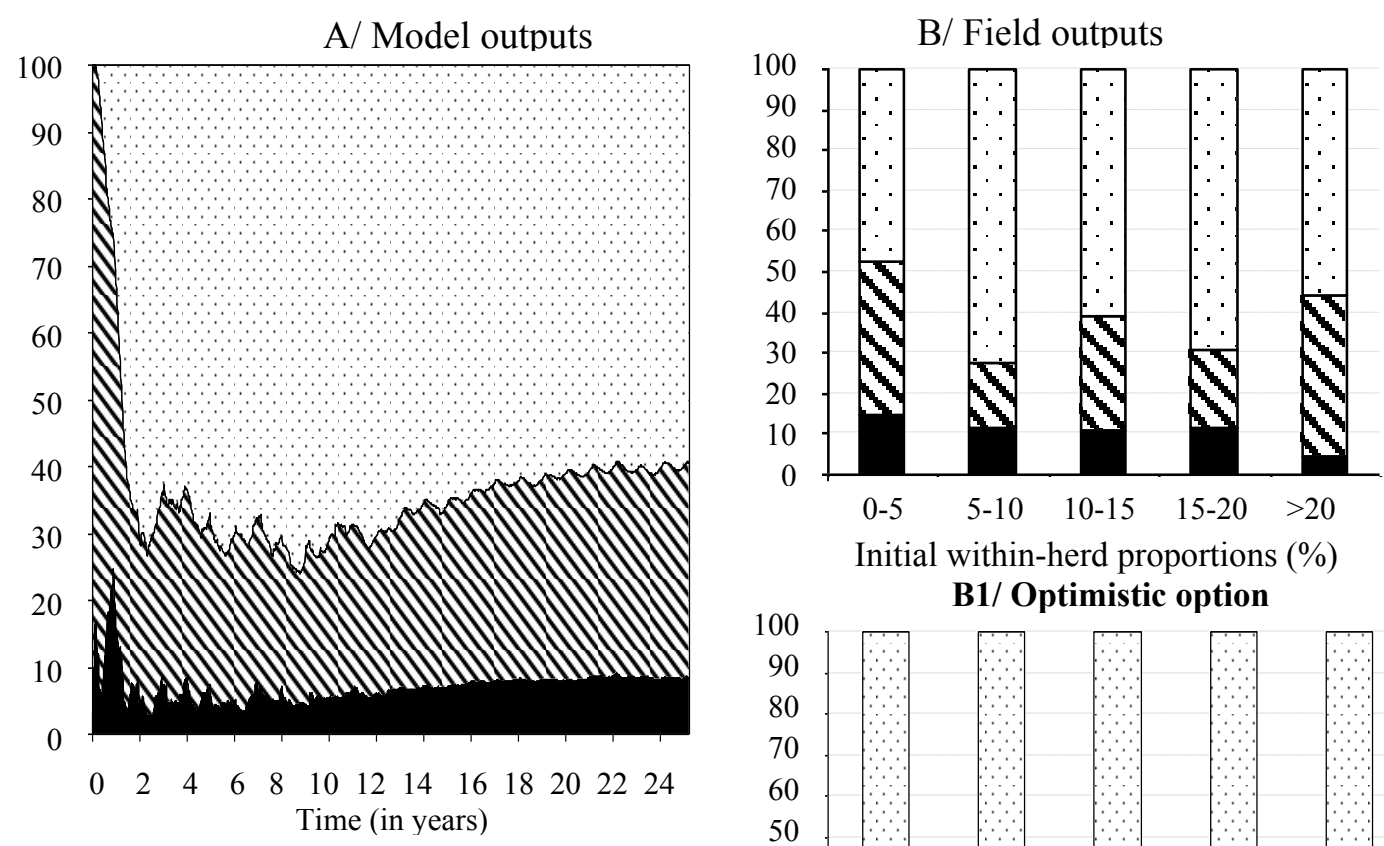

Initial within-herd proportions (\%)

B1/ Optimistic option

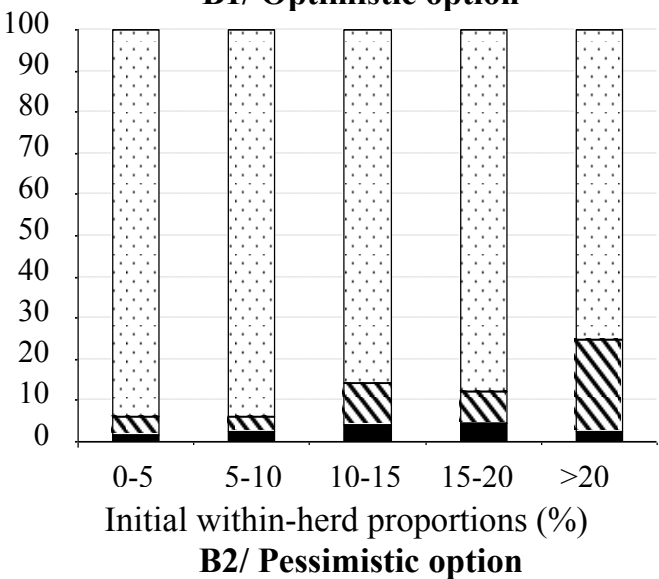

Figure 6 Comparison of the simulated and observed distributions of Mycobacterium avium subsp. paratuberculosis (Map) infectedadults perinfection state in infected dairy cattle herds. A/Simulated mean distribution over time in persistently infected herds as predicted by a Map spread model within a dairy cattle herd; B/Mean percentage of tested adults per infection states based on individual life long determined status in 59 herds at enrolment in a paratuberculosis control program in France, before any control measure is implemented, according to the range of the initial within-herd prevalence. Animals tested twice or less and having negative results are assumed to be either resistant (state not shown) (B1: optimistic option) or latently infected (B2: pessimistic option).

Map infection dynamics towards persistence. Furthermore, it is very easy to use in the field. A threshold of two affected cows seems adequate to trigger control measures in a herd. However, a farmer may miss the $1^{\text {st }}$ clinical case and be unaware that there already have been two cases in his herd. An earlier indicator would be useful. Combining clinical surveillance with an imperfect test implemented on all potentially infected adults could reduce the time needed for detection. In that case, a threshold of three detected animals seems adequate. To assess the economic advantage of such surveillance, both the costs and benefits of early detection need to be analyzed.

In the absence of control measures, the simulated mean prevalence in infected cattle increased to $88 \%$ after 25 years in the model, as previously published models also have shown [3-5,23]. These levels of prevalence are not expected with field data as control measures will be implemented long before such levels are reached. However, herds with high apparent prevalence are found, which corresponds to these levels of true prevalence (e.g. $[17,24,25])$. Moreover, simulated prevalence between 5 and 9 years after Map introduction was lower than levels observed on farms prior to enrolment in a control programme. This suggests that the range of observed prevalence at control programme enrolment typically corresponds to a more advanced stage of within-herd Map dynamics, when without any control measure fadeout would rarely occur.

With this new model, it was possible to assess the relative importance of transmission routes on Map spread in a dairy herd. This model accounts not only for vertical transmission and horizontal transmission via the ingestion of Map in milk and colostrum, as has been done in previously published models [6], but also for indirect contacts between animals of different ages 


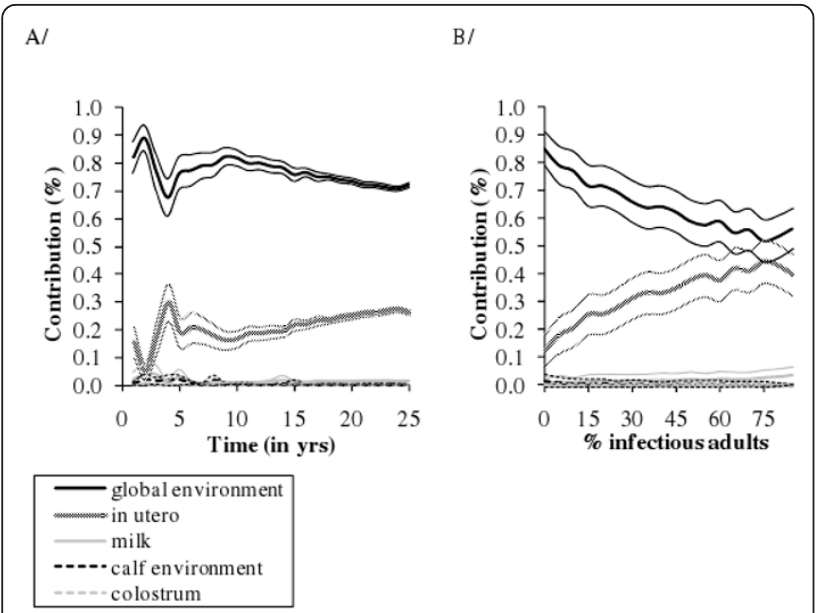

Figure 7 Mean relative contributions of the 5 transmission routes of Mycobacterium avium subsp. paratuberculosis (Map) infection in persistently infected dairy cattle herds (118 runs out of 400 ). A/over time since Map introduction in the herd; B/over prevalence of infectious adults. Map is introduced only once $(t=0)$.

raised in different groups, and horizontal transmission via the ingestion of faeces present in the contaminated environment. Possible exposure of calves to adults or to other calves is modelled and the level of exposure varies depending on calf age and calf housing facilities. In persistently infected herds, contamination of the environment by adults was the main transmission route, in utero transmission being the second. Calf-to-calf transmission appeared to be a minor route of transmission. However, in this model, milk and colostrum routes of transmission correspond to liquid contamination by the dam (direct shedding or faecal contamination), not contamination through the environment. On the other hand, possible faecal contamination of buckets used to give milk to calves is considered to be an element of global environmental contamination, not the milk route of transmission. As a priority, exposure of calves to any environment contaminated by adult faeces should be reduced, particularly at and just after birth when calves are the most susceptible.

The model has been evaluated and provides qualitative predictions such as ranking routes and the description of possible dynamics. The model validation has been performed by comparing model outputs with field data. A hypothesis-testing approach has been used allowing us to conclude that our findings are robust to variation in uncertain model parameters. For some of the uncertain parameters $\left(h, p_{L}, \beta_{g}\right)$, the true value is likely to be within a smaller interval than $\pm 50 \%$ of their reference value as larger variations led to results inconsistent with observations. However, only a partial validation has been possible because the introduction date of Map into a herd was not known for the observed field data. Furthermore, we assumed here herds are closed (a single Map introduction), whereas data may concern open herds with multiple introduction of potentially infected cattle. Finally, in practice, when paratuberculosis is diagnosed, farmers are likely to change their routines to ensure their animals' welfare and protect their economic interests. It would be unethical to recommend that they do nothing. In contrast, we can model herds in which no control measures are implemented.

In the model, we neglected some processes and factors that may interfere with Map spread but that are not yet sufficiently described. First, we did not represent passive or intermittent shedding in the model. The intermittent shedding sometimes noticed [26] indeed could be explained by the low sensitivity of diagnostic tests or by heterogeneity of faeces or milk samplings [27] which

Table 4 Proportion (\%) of runs having 0 to more than 3 clinically affected and/or subclinically infected animals (Is) detected (sensitivity of $\mathbf{0 . 5}$ and specificity of $\mathbf{1}$ for the tests used for Is animals detection) after $\mathbf{1}$ to 5 years of simulation in herds with spontaneous fadeout or persistent infection

\begin{tabular}{|c|c|c|c|c|c|c|c|c|c|c|c|}
\hline & \multirow[t]{3}{*}{ Cumulated number of animals $(n r)$} & \multicolumn{5}{|c|}{$\begin{array}{c}\% \text { of runs with } n r \\
\text { clinically affected } \\
\text { animals }\end{array}$} & \multicolumn{5}{|c|}{$\begin{array}{c}\% \text { of runs with } n r \\
\text { clinically affected \& } \\
\text { detected subclinically } \\
\text { infected animals }\end{array}$} \\
\hline & & \multicolumn{5}{|c|}{ Time (in years) } & \multicolumn{5}{|c|}{ Time (in years) } \\
\hline & & 1 & 2 & 3 & 4 & 5 & 1 & 2 & 3 & 4 & 5 \\
\hline \multirow[t]{4}{*}{ Proportion among herds with fadeout (282 runs) } & 0 & 75 & 67 & 64 & 62 & 62 & 40 & 37 & 37 & 35 & 35 \\
\hline & 1 & 25 & 33 & 36 & 36 & 35 & 50 & 48 & 45 & 45 & 44 \\
\hline & 2 & 0 & 0 & 0 & 1 & 2 & 10 & 14 & 17 & 17 & 17 \\
\hline & $\geq 3$ & 0 & 0 & 0 & 1 & 1 & 0 & 0 & 1 & 3 & 4 \\
\hline \multirow[t]{4}{*}{ Proportion among persistently infected herds (118 runs) } & 0 & 48 & 23 & 9 & 5 & 2 & 24 & 8 & 2 & 1 & 1 \\
\hline & 1 & 52 & 75 & 67 & 40 & 18 & 46 & 51 & 30 & 14 & 3 \\
\hline & 2 & 0 & 2 & 15 & 15 & 19 & 60 & 34 & 25 & 22 & 8 \\
\hline & $\geq 3$ & 0 & 0 & 9 & 40 & 61 & 0 & 7 & 43 & 63 & 88 \\
\hline
\end{tabular}


lead to an intermittent detection of infectious animals. If such intermittent shedding were to be shown, a different modelling approach would have to be used, where a given probability of shedding in the latent state according to age or to the physiological status (in gestation, in lactation, etc) would have to be assumed. However, given the current knowledge such a model cannot be parameterized. Moreover, such intermittent shedders would not be directly in contact with susceptible calves but be raised together with other adults. Their contribution to the global environmental contamination thus would be very limited as it would be diluted by the quantity of Map shed by subclinically infected and clinically affected animals. Therefore, the environmental contamination would be only slightly higher assuming latently infected animals shed intermittently. Second, super-shedders have been described $[28,29]$ but it is unknown whether they are specific animals or if shedding of all infectious animals varies highly over time. Therefore, we assumed here any animal can shed a high amount of Map at random time. Third, experimental animal models suggest there could be genetic factors responsible for resistance or susceptibility to Map infection [30]. Several genes have been identified to date. However, current knowledge is insufficient to include such genetic factors in modelling. Lastly, the incubation period is inversely related to the challenge dose, clinical signs occurring sooner under experimental than natural conditions [31]. However, the mechanism of the doseresponse effect, the potential cumulative exposure, and the minimum infection dose are still uncertain. Therefore, this has not been included in the model.

The model could be adapted to open dairy herds and used to evaluate control measures in both open and closed herds. Furthermore, this model could be used for herds of different sizes having similar herd structure and management. Herd management is driven by a number of parameters which gives flexibility to the model. However, the model would need to be modified if the structure of the herd is markedly different as exposure to the contaminated environment would differ.

\section{Acknowledgements}

The authors gratefully acknowledge A Lehebel and D Taisne for data analysis. Financial support for this research was provided by the French National Institute for Agricultural Research, Cemagref and Basse-Normandie, Bretagne, Pays de le Loire et Poitou-Charentes Regional Councils under SANCRE project, in the framework of "For and About Regional Development" programs, and by the French Ministry of Agriculture.

\section{Author details}

'INRA, UMR1300 Bio-agression, Epidémiologie et Analyse de Risque en santé animale, BP 40706, 44307 Nantes, France. ${ }^{2}$ ONIRIS, UNAM Université Nantes Angers Le Mans, France. ${ }^{3}$ Veterinary Epidemiology \& Public Health Division, Royal Veterinary College, University of London, Hatfield AL9 7TA, Hertfordshire, UK.

\section{Authors' contributions}

$\mathrm{CM}$ and PE conceived of the study, carried out the model development and analysis, and drafted the manuscript. CF conceived of the study, participated in its design and coordination, and drafted the manuscript. HS conceived of the study and participated in its design. DP participated in the study design and coordination. All authors read, amended and approved the final manuscript.

\section{Competing interests}

The authors declare that they have no competing interests.

Received: 2 July 2010 Accepted: 15 February 2011

Published: 15 February 2011

\section{References}

1. Weber MF, Verhoeff J, van Schaik G, van Maanen C: Evaluation of ZiehlNeelsen stained faecal smear and ELISA as tools for surveillance of clinical paratuberculosis in cattle in the Netherlands. Prev Vet Med 2009, 92:265-266.

2. Nielsen SS: Transitions in diagnostic tests used for detection of Mycobacterium avium subsp. paratuberculosis infections in cattle. Vet Microbiol 2008, 132:274-282.

3. Groenendaal H, Nielen M, Jalvingh AW, Horst SH, Galligan DT, Hesselink JW: A simulation of Johne's disease control. Prev Vet Med 2002, 54:225-245.

4. Kudahl AB, Ostergaard S, Sorensen JT, Nielsen SS: A stochastic model simulating paratuberculosis in a dairy herd. Prev Vet Med 2007, 78:97-117.

5. Pouillot R, Dufour B, Durand B: A deterministic and stochastic simulation model for intra-herd paratuberculosis transmission. Vet Res 2004, 35:53-68.

6. Marcé C, Ezanno P, Seegers H, Pfeiffer DU, Fourichon C: Modeling withinherd transmission of Mycobacterium avium paratuberculosis in dairy cattle: a review. J Dairy Sci 2010, 93:4455-4470.

7. Jorgensen JB: Survival of Mycobacterium paratuberculosis in slurry. Nord Vet Med 1977, 29:267-270.

8. Whittington RJ, Marshall DJ, Nicholls PJ, Marsh IB, Reddacliff LA: Survival and dormancy of Mycobacterium avium subsp. paratuberculosis in the environment. Appl Environ Microbiol 2004, 70:2989-3004.

9. Van Roermund HJW, Bakker D, Willemsen PTJ, De Jong MCM: Horizontal transmission of Mycobacterium avium subsp. paratuberculosis in cattle in an experimental setting: Calves can transmit the infection to other calves. Vet Microbiol 2007, 122:270-279.

10. Ezanno P, Fourichon C, Beaudeau F, Seegers H: Between-herd movements of cattle as a tool for evaluating the risk of introducing infected animals. Anim Res 2006, 55:189-208.

11. Ezanno P, van Schaik G, Weber MF, Heesterbeek JA: A modeling study on the sustainability of a certification-and-monitoring program for paratuberculosis in cattle. Vet Res 2005, 36:811-826.

12. The Scilab website. [http://www.scilab.org].

13. Marcé C, Guatteo R, Bareille N, Fourichon C: Dairy calf housing systems across Europe and risk for calf infectious diseases. Animal 2010, 4:1588-1596.

14. Windsor PA, Whittington RJ: Evidence for age susceptibility of cattle to Johne's disease. Vet J 2010, 184:37-44.

15. Hagan WA: Age as a factor in susceptibility to Johne's disease. Cornell Vet $1938,28: 34-40$

16. Nielsen SS, Ersboll AK: Age at occurrence of Mycobacterium avium subspecies paratuberculosis in naturally infected dairy cows. J Dairy Sci 2006, 89:4557-4566.

17. Weber MF, Kogut J, de Bree J, van Schaik G, Nielen M: Age at which dairy cattle become Mycobacterium avium subsp. paratuberculosis faecal culture positive. Prev Vet Med 2010, 97:29-36.

18. Whitlock RH, Wells SJ, Sweeney RW, Van Tiem J: ELISA and fecal culture for paratuberculosis (Johne's disease): sensitivity and specificity of each method. Vet Microbiol 2000, 77.378-398.

19. Nielsen SS, Toft N: Ante mortem diagnosis of paratuberculosis: A review of accuracies of ELISA, interferon- $\gamma$ assay and faecal culture techniques. Vet Microbiol 2008, 129:217-235

20. Guicharnaud M: Prevalence of paratuberculosis in dairy cattle herd worldwide: review and analysis. Doctorate in Veterinary Medicine Thesis Nantes, France; 2009, (in French). 
21. Nielsen SS, Toft N: A review of prevalences of paratuberculosis in farmed animals in Europe. Prev Vet Med 2009, 88:1-14

22. Taisne D: A control programme for paratuberculosis in infected dairy herds in the Ille-et-Vilaine county. Doctorate in Veterinary Medicine Thesis Nantes, France; 2009, (in French).

23. Mitchell RM, Whitlock RH, Stehman SM, Benedictus A, Chapagain PP Grohn YT, Schukken YH: Simulation modeling to evaluate the persistence of Mycobacterium avium subsp. paratuberculosis (MAP) on commercial dairy farms in the United States. Prev Vet Med 2008, 83:360-380.

24. Benedictus A, Mitchell RM, Linde-Widmann M, Sweeney R, Fyock T, Schukken YH, Whitlock RH: Transmission parameters of Mycobacterium avium subspecies paratuberculosis infections in a dairy herd going through a control program. Prev Vet Med 2008, 83:215-227.

25. Woodbine KA, Schukken YH, Green LE, Ramirez-Villaescusa A, Mason S, Moore SJ, Bilbao C, Swann N, Medley GF: Seroprevalence and epidemiological characteristics of Mycobacterium avium subsp. paratuberculosis on 114 cattle farms in south west England. Prev Vet Med 2009, 89:102-109.

26. Whittington RJ, Reddacliff LA, Marsh IB, McAllistair S, Saunders V: Temporal patterns and quantification of excretion of Mycobacterium avium subsp paratuberculosis in sheep with Johne's disease. Aust Vet J 2000, 78:34-37.

27. Pradhan AK, Kramer AJ, Mitchell RM, Whitlock RH, Smith JM, Hovingh E, Van Kessel JS, Karns JS, Schukken YH: Multilocus short sequence repeat analysis of Mycobacterium avium subsp. paratuberculosis isolates from dairy herds in Northeastern United States of a longitudinal study indicates low shedders are truly infected. Proc. of the 10th International Colloquium on Paratuberculosis Minneapolis, USA; 2009, 30-33.

28. Hovingh E, Whitlock R, Sweeney R, Fyock T, Wolfgang D, Smith J, Schukken $Y$ : Identification and implications of Map supershedders. Joint meeting of the ADSA, AMSA, ASAS and PSA Minneapolis, MN; 2006.

29. Whitlock RH, Sweeney RW, Fyock TL, Smith J: MAP Super-Shedders: Another factor in the control of Johne's disease. Proc. of the 8th International Colloquium on Paratuberculosis Copenhagen, Denmark; 2005, 42.

30. Koets AP, Adugna G, Janss LLG, Van WHJ, Kalis CHJ, Wentink GH, Rutten VPMG, Schukken YH: Genetic variation of susceptibility to Mycobacterium avium subsp. paratuberculosis infection in dairy cattle. J Dairy Sci 2000, 83:2702-2708.

31. Whittington RJ, Sergeant ESG: Progress towards understanding the spread, detection and control of Mycobacterium avium subsp paratuberculosis in animal populations. Aust Vet J 2001, 79:267-278.

32. Rio O: Frequency and risks of mortality and health disorders of calves in dairy cattle herds. Doctorate in Veterinary Medicine Thesis Nantes, France; 1999, (in French).

33. Jégou $V$, Porhiel JY, Brunschwig P: Risk management factors affecting mortality among dairy calves herds in 80 herds in Brittany. Proc. Journées Bovines Nantaises Nantes, France; 2006, 6, (in French).

34. Beaudeau F: Review of culling and replacement practices in dairy cattle herds. Master Thesis Institut Supérieur des Productions Animales, Rennes, France; 1991, (in French).

35. Whittington RJ, Windsor PA: In utero infection of cattle with Mycobacterium avium subsp. paratuberculosis: A critical review and meta-analysis. Vet J 2009, 179:60-69.

36. Whitlock $\mathrm{RH}$, Buergelt C: Preclinical and clinical manifestations of paratuberculosis (including pathology). Vet Clin North Am Food Anim Pract 1996, 12:345-356.

37. Matthews HT: On Johne's disease. Vet Rec 1947, 59:397-401.

38. Streeter RN, Hoffsis GF, Bech-Nielsen S, Shulaw WP, Rings M: Isolation of Mycobacterium paratuberculosis from colostrum and milk of subclinically infected cows. Am J Vet Res 1995, 56:1322-1324.

39. Sweeney RW, Whitlock RH, Rosenberger AE: Mycobacterium paratuberculosis isolated from fetuses of infected cows not manifesting signs of the disease. Am J Vet Res 1992, 53:477-480.

40. Begg DJ, Whittington RJ: Experimental animal infection models for Johne's disease, an infectious enteropathy caused by Mycobacterium avium subsp. paratuberculosis. Vet J 2008, 176:129-145.

41. Nielsen SS, Enevoldsen C, Toft N: Milk production losses associated with bovine paratuberculosis diagnosed from repeated testing. Proc. 11th International Symposia of Veterinary Epidemiology and Economics Cairns, Australia; 2006, 619.
42. Giese SB, Ahrens P: Detection of Mycobacterium avium subsp. paratuberculosis in milk from clinically affected cows by PCR and culture. Vet Microbiol 2000, 77:291-297.

43. Magnusson M, Christiansson A, Svensson B, Kolstrup C: Effect of different premilking manual teat-cleaning methods on bacterial spores in milk. J Dairy Sci 2006, 89:3866-3875.

44. Vissers MMM, Driehuis F, Te Giffel MC, De Jong P, Lankveld JMG: Improving farm management by modeling the contamination of farm tank milk with butyric acid bacteria. J Dairy Sci 2006, 89:850-858.

45. Rossiter CA, Burhans WS: Farm-specific approach to paratuberculosis (Johne's disease) control. Vet Clin North Am Food Anim Pract 1996, 12:383-415.

46. Jorgensen JB: An improved medium for culture of Mycobacterium paratuberculosis from bovine faeces. Acta Vet Scand 1982, 23:325-335.

doi:10.1186/1297-9716-42-36

Cite this article as: Marcé et al:: Predicting fadeout versus persistence of paratuberculosis in a dairy cattle herd for management and control purposes: a modelling study. Veterinary Research 2011 42:36.

\section{Submit your next manuscript to BioMed Central and take full advantage of:}

- Convenient online submission

- Thorough peer review

- No space constraints or color figure charges

- Immediate publication on acceptance

- Inclusion in PubMed, CAS, Scopus and Google Scholar

- Research which is freely available for redistribution

Submit your manuscript at www.biomedcentral.com/submit
C) Biomed Central 